\title{
Paper Modeling and Analysis of Additional Clear Channel Assessment to Improve Performance of IEEE 802.15.4-based MAC Protocol
}

\author{
Manjunath G. Asuti and Prabhugoud I. Basarkod \\ School of Electronics and Communication Engineering, REVA University, Bengaluru, India
}

https://doi.org/10.26636/jtit.2020.140420

\begin{abstract}
Design of the MAC protocol is crucial in all wireless sensor networks (WSNs) due to its influence on the performance of the transceiver, i.e. the most energy-consuming component of each sensor node. A mechanism known as "carrier sense multiple access with collision avoidance" (CSMA/CA) is used for accessing the wireless channel in the IEEE 802.15.4 standard-based MAC protocol in order to avoid collisions between the network's communicating nodes. CSMA/CA relies on two clear channel assessments $(\mathrm{CCA}=2)$ for checking the status of the channel. In this paper, we develop an additional CCA algorithm for the two scenarios encountered in star topology-enabled WSNs. Next, we investigate the impact of an additional clear channel assessment $(\mathrm{CCA}=3)$ on performance in IEEE 802.15.4. We develop a Markov chain model for the proposed methodology, and validate it using Matlab. Simulation results show that there is a significant improvement of performance metrics in the IEEE 802.15.4 standard-based $\mathrm{MAC}$ protocol with an additional CCA.
\end{abstract}

Keywords-clear channel assessment, CSMA/CA mechanism, IEEE 802.15.4, MAC protocol, wireless sensor networks.

\section{Introduction}

Short range, low rate communication systems, such as low rate wireless personal area networks (LR-WPANs) find various applications in smart meters, industrial sensing, health care, home automation and monitoring. The IEEE standard-based definition used for low rate transmissions describes the physical and MAC layers of wireless sensor networks. The MAC layer that complies with the IEEE 802.15.4 standard [1]-[5] uses the "carrier sense multiple access with collision avoidance" (CSMA/CA) mechanism for accessing the channel. Current research focuses on the improvement of performance parameters of IEEE 802.15.4based MAC protocols, in order to achieve different objectives, thus enabling numerous WSN applications.

The standard CSMA/CA mechanism accesses the wireless channel (by checking its status), relying on clear channel assessment $(\mathrm{CCA})$ with a standard value $=2$. The status of the channel is checked twice to determine whether the medium is busy or idle, before commencing data transmis- sion. Later on, based on the condition of the channel, the nodes will transmit data in the medium in order to avoid the probability of a collision within the network. Furthermore, CCA may be either of the single or double variety, depending on the periodic traffic pattern in the network [6]. IEEE 802.15.4-based WSNs may be based either on star or peer-to-peer topologies, depending on the applicationrelated requirements. In star topologies, a personal area network (PAN) coordinator is necessary to establish communication between any pair of nodes.

In order to maintain better synchronization between the communicating nodes, the PAN coordinator adopts a superframe structure which specifies beacon interval (BI), active period, superframe duration and inactive period. The beacon signal is used for synchronization of the frames sent by the PAN coordinator to other nodes. BI constitutes the period estimated between the start and the end of a given frame. Therefore, $B I=$ aBaseSuperframeduration $\times 2^{B O}$ symbols $(B O \leq 14)$. Active period is specified by the superframe duration parameter, given by $S D=$ aBaseSuperframeduration $\times 2^{S O}$ symbols $(0 \leq S O \leq B O$ $\leq 14)$. The BO attribute determines the beacon order and SO specifies superframe duration, i.e. the active part of the superframe structure.

The active period consists of 16 slots which are subdivided into contention access period (CAP) and contention free period (CFP). Under CAP, the nodes will utilize the slotted CSMA/CA CSMA/CA mechanism and the PAN coordina-

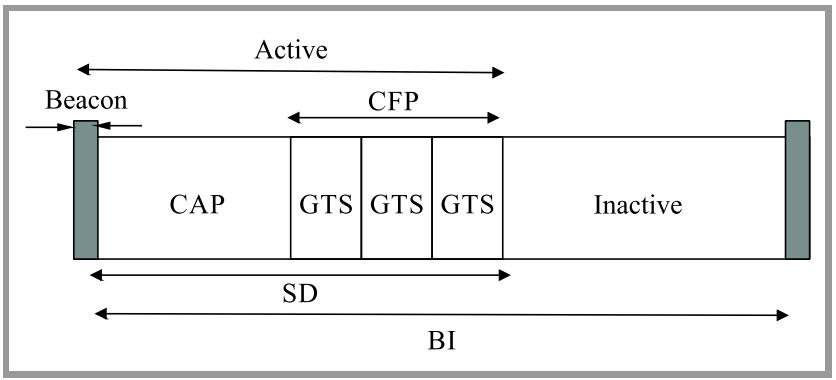

Fig. 1. Superframe structure of the IEEE 802.15.4 standard. 
tor plays a major role during CFP in which the nodes send requests for guaranteed time slots (GTS) to the PAN coordinator. The inactive period provides details of the node in sleep state. Figure 1 illustrates the superframe structure utilized for the standard CSMA/CA mechanism.

\subsection{CSMA/CA Standard}

The CSMA/CA algorithm operates in two modes, namely beacon-enabled mode and non-beacon-enabled mode. Here, the beacon-enabled mode of the CSMA/CA mechanism is considered for channel assessment purposes.

The standard initializes three parameters, i.e. number of backoff stages $(\mathrm{NB}=0)$, contention window $(\mathrm{CW}=2)$, and minimum value of backoff exponent $\left(\mathrm{BE}_{\mathrm{min}}\right)$. Backoff time is initialized for setting the delay in the range of $2^{\mathrm{BE}}-1$. The backoff period varies from $\mathrm{BE}_{\min }$ to the max value of backoff exponent $\left(\mathrm{BE}_{\max }\right)$. CCA is performed for checking the status of the channel.

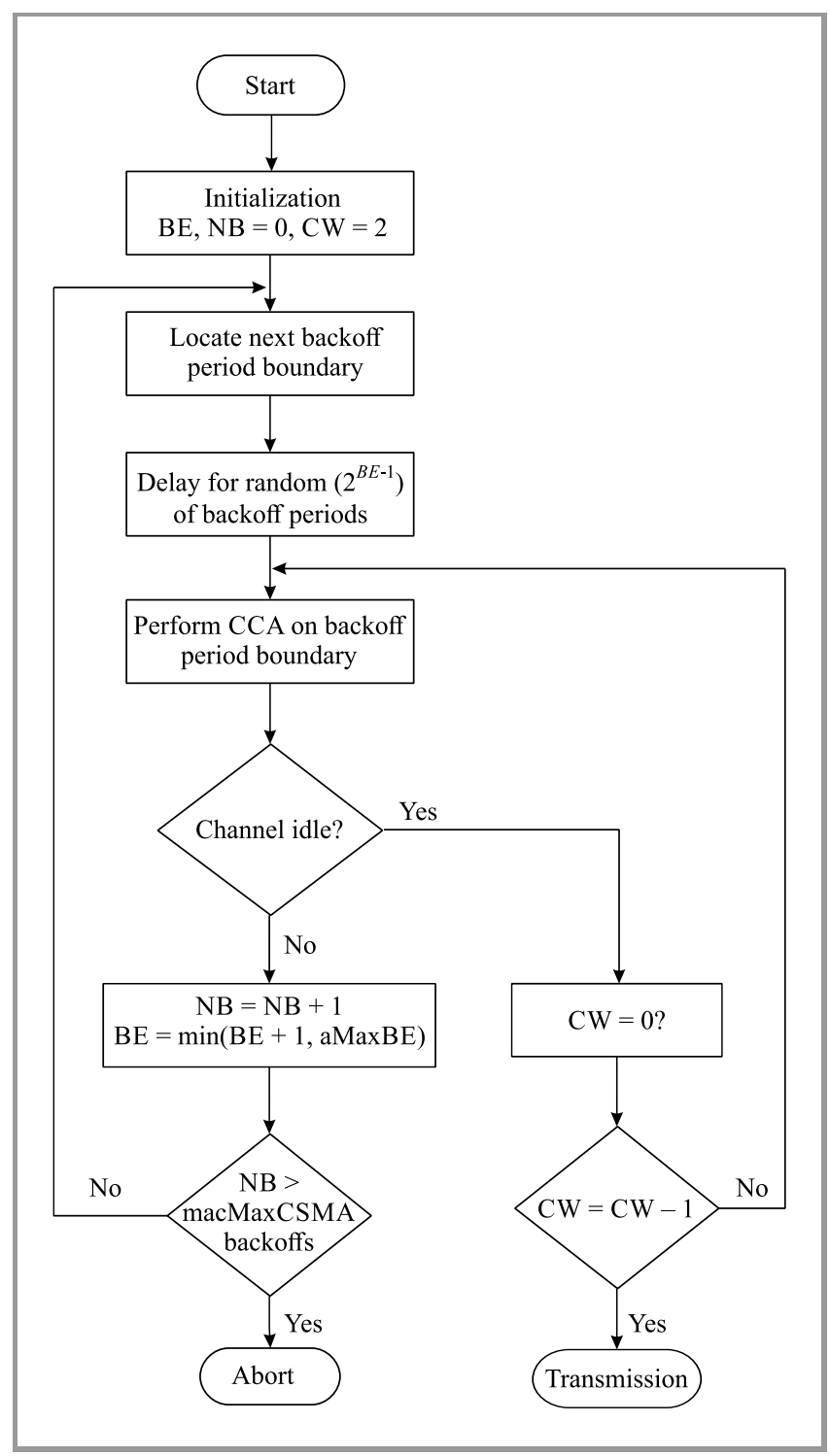

Fig. 2. CSMA/CA mechanism compliant with the IEEE 802.15.4 standard.
Upon identifying that the channel is busy, NB and BE are incremented by 1 , and $\mathrm{CW}$ remains unchanged $(\mathrm{CW}=2)$. If the CSMA backoffs exceed the limit, the frames are dropped. Otherwise, the algorithm proceeds to the next. Upon determining that the channel status is free, $\mathrm{CW}$ is decremented by 1 . When $\mathrm{CW}=0$, the data frame is transmitted by the node.

Figure 2 shows the CSMA/CA mechanism defined for the IEEE 802.15.4 standard. CSMA/CA adapts the number of CCAs (standard value $=2$ ) to verify the status of the channel. However, in the existing CSMA/CA mechanism, some of the performance metrics are degraded for certain traffic scenarios. We need to address the issues and problems encountered in the CSMA/CA mechanism used in the IEEE 802.15.4 standard-based MAC protocol.

\subsection{Research Contribution}

In this paper, the impact of conducting an additional CCA covering some of the performance metrics of IEEE 802.15.4-based MAC protocol is investigated. The main contributions of this paper are summarized as follows:

- An accurate Markov chain model for the proposed additional CCA is developed to improve performance of IEEE 802.15.4-based MAC protocol. The proposed model is based on two scenarios encountered in the network due to two successive CCA (standard value $=2$ ) failures.

- Based on the proposed model, the impact of the additional CCA on the performance metrics in the IEEE 802.15.4 standard is investigated.

- The performance of the additional CCA is evaluated based on extensive simulations performed using Matlab. Additionally, the proposed methodology is compared with the existing CCA approaches. Finally, the paper shows that by incorporating an additional $\mathrm{CCA}$, it is possible to improve performance metrics of the IEEE 802.15.4 standard.

In Section 2, related work is presented concerning various CCA approaches introduced in the IEEE 802.15.4 standard. In Section 3, the problem is described for two scenarios considered in a star topology network. In Section 4, the proposed additional CCA algorithm is presented. In Section 5, the Markov chain model for the proposed additional CCA is shown. In Section 6, various performance metrics are developed and analyzed. In Section 7, performance evaluation is performed for the additional CCA and the results obtained are compared with the IEEE 802.15.4-based MAC protocol. Section 8 concludes the paper.

\section{Related Work}

In paper [7], the authors presented an additional carrier sensing algorithm for channel access mechanism in the IEEE 802.15.4 standard, improving overall throughput, power consumption, and MAC delay. In article [8], the authors studied the effect of CCA-related variation con- 
duction on some of the performance metrics of the IEEE 802.15.4-based MAC protocol. Paper [9] proposed a new backoff scheme for the standard with an improvement in throughput and energy efficiency.

In [10], the authors adopted a new channel access mechanism referred to as the linearly increased backoff (LIB) method, with an enhancement of packet delay, throughput, and energy consumption. Authors of [11] developed a second chance CCA algorithm which consumes less energy and decreases the average delay required to conduct CCA. In [12], a channel access mechanism for the shared link existing between two or more devices in a time-slotted channel hopping (TSCH) network is presented. A model for performance metrics, such as average access delay, throughput, and packet loss rate is developed. In paper [13], the authors observed a variation in MAC parameters with fading channel conditions in IEEE 802.15.4. They argue that fading channel conditions positively affected power consumption and the number of packets received successfully in the network. The authors of [14] showed a new wireless channel access mechanism which improved reliability and reduced energy consumption of the node.

Article [15] shows a new approach to accessing channels, obtaining the precise status of a channel for packet transmission. The authors have proven that throughput increased by $8.76 \%$ and decreased the number of CCAs required by $3.9 \%$. Paper [16] presents an efficient blind adaptive access parameter tuning algorithm which consumes less energy and improves the reliability of IEEE networks. The algorithm is based on reducing the extra overhead required for the backoff process without acquiring the acknowledgement signal. In [17], the authors developed a new channel access mechanism for IEEE 802.15.4 networks, providing channel access to all devices with a high buffer capacity, offering a minimum packet drop rate and high throughput. In article [18], the authors presented a new CSMA/CA mechanism based on different priorities assigned to the nodes, i.e. they came up with a classification of nodes based on three different priorities, such as fixed channel access for nodes with priority- 0 and different probabilities for nodes with priority- 1 and priority- 2 . The authors of [19] analyzed an increase in CSMA waiting time with specific MAC parameters being varied under different traffic conditions. They claimed that the collision probability was reduced when the macminBE value is increased. Article [20] shows a contention differentiated adaptive slot allocation CSMA/CA algorithm for wireless body area networks, reducing collision probability by minimizing packet retransmission.

In paper [21], a real-time scenario used in wireless body area networks for tuning some of the MAC parameters adaptively, in accordance with the IEEE 802.15.4 standard, as well as for maximizing the packet delivery ratio and reliability of the network is considered. In [22], a novel adaptive duty cycle algorithm for channel access mechanism in IEEE 802.15.4 networks, with adjustment of the duty cycle to different traffic conditions is proposed. It is proved that energy efficiency is improved for high traffic conditions. In [23], the authors proposed an enhanced CCA performed in accordance with the IEEE 802.15.4 standard, which increased throughput by $7 \%$.

In article [24], a new method for the backoff mechanism used in IEEE 802.15.4 is presented. According to the authors, the backoff period is subdivided into two backoffs, i.e. temporary backoff and next temporary backoff. They claim that the probability of a collision is minimized, and that throughput and packet delivery ratio are improved. In [25], the authors designed an efficient CSMA/CA algorithm for IEEE 802.15.4 networks, utilizing the variable backoff exponent to increase efficiency of data transmission, thereby reducing the packet drop rate.

Paper [26] proposed a new backoff mechanism called priority-based binary exponential backoff (PB-BEB), in which the number of CCAs performed is not equal to two. PB-BEB varied the number of CCAs required for channel access, depending on the number of backoffs undergone by the node, i.e. the highest priority is assigned to the node which has undergone more backoffs during earlier time slots while accessing the wireless channel. The authors claim that PB-BEB outperforms the binary exponential backoff method (BEB) employed in the IEEE 802.15.4 standard.

\section{Problem Statement}

Let us consider scenario 1 with a star topology-enabled WSN, with $N_{T}$ sensor devices located within the transmission range of a PAN coordinator node. Let A and B denote two devices contending for channel access within the network. At time slot $t_{1}$, let device A be successful in conducting the first CCA (upon finding the idle channel). At time slot $t_{2}$, device A finds the channel busy, after conducting the second CCA. Alternatively, at the same time slot $t_{2}$, device $\mathrm{B}$ conducts the first CCA. In this scenario, the IEEE 802.15.4 standard $(\mathrm{CCA}=2)$ fails, because device A finds the channel busy while conducting the second CCA. Figure 3 presents scenario 1 considered for the additional CCA.

Scenario 2 considers a star topology-enabled WSN with $N_{T}$ sensor devices located within the transmission range of a PAN coordinator node. At time slot $t_{1}$, let device A be successful in conducting the first CCA (upon finding the idle channel). At time slot $t_{2}$, device A finds the channel busy, after conducting the second CCA. Alternatively, at the same time slot $t_{2}$, device B waits for an acknowledgement signal from the PAN coordinator node. In this scenario, the IEEE 802.15.4 standard $(\mathrm{CCA}=2)$ fails, because device A finds the channel busy while conducting the second CCA. Figure 4 presents scenario 2 considered for the additional CCA.

\section{Proposed Methodology}

Here, a detailed explanation of the proposed additional CCA algorithm is provided for the two scenarios encoun- 


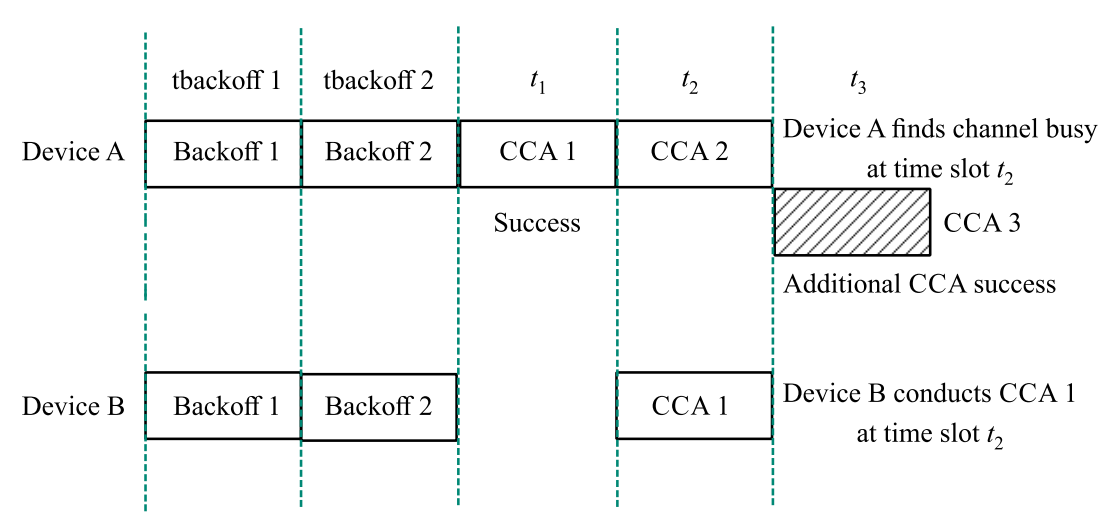

Fig. 3. Scenario 1 considered for additional CCA.

\begin{tabular}{|c|c|c|c|c|c|}
\hline \multirow{4}{*}{ Device A } & tbackoff 1 & tbackoff 2 & $t_{1}$ & $t_{2}$ & \multirow{2}{*}{$\begin{array}{l}t_{3} \\
\text { Device A finds channel busy } \\
\text { at time slot } t_{2}\end{array}$} \\
\hline & Backoff 1 & Backoff 2 & CCA 1 & CCA 2 & \\
\hline & & & Success & & $2 / 1 / 1 / 1 / 20 \begin{array}{c}\text { Data } \\
\text { transmitted }\end{array}$ \\
\hline & & & & & Additional CCA success \\
\hline \multirow[t]{2}{*}{ Device B } & Backoff 1 & Backoff 2 & & $t_{A c k}$ & \multirow{2}{*}{$\begin{array}{l}\text { Device B waits for Ack from } \\
\text { PAN coordinator slot } t_{2}\end{array}$} \\
\hline & & & & & \\
\hline
\end{tabular}

Fig. 4. Scenario 2 considered for additional CCA.

tered in a star topology network. Algorithm 1 is incorporated to enhance performance of the IEEE 802.15.4-based MAC protocol. It assumes that two nodes in a network are contending for a channel and that the highest priority is assigned to the node which has completed multiple backoffs, rather than to the node which has just started to conduct CCA.

The algorithm starts by initializing inputs contention window $(\mathrm{CW}=3)$, number of backoff stages $(\mathrm{NB}=0)$, minimum value of backoff exponent (macMinBE=3), maximum value of backoff exponent (macMaxBE=5), and the number of sensor devices (line 1). Additionally, the time slots required to complete the backoffs and the CCA states (line 2) are determined. Next, the channel access mechanism is set by locating the backoff period boundary (lines 5 and 6). Therefore, it waits for a random period $\left(0\right.$ to $\left.2^{B E}-1\right)$ for certain backoff time slots. The number of backoff time slots is $t_{\text {backoff } 1}, t_{\text {backoff } 2}, \ldots, t_{\text {backoffn }}$ (line 7 ).

After a suitable backoff period, at time slot $t_{1}$, Node_A performs CCA_1 to check the status of the channel (line 8). Then, the algorithm enters into a loop, checking the status of the channel, and determining it to be either idle or busy. Next, if the channel is found to be idle at the end of its first CCA, the contention window is decremented until it reaches zero $(\mathrm{CW}=0)$ (line 9). Otherwise, the algorithm jumps to line 15 (where the channel is busy). However, if
$\mathrm{CW}=0$, then Node_A is set to transmit a data packet and waits for an acknowledgement signal in the network (line 10). Otherwise, CCA_1 expires and the algorithm moves to line 13 . Here, if the channel is found to be busy after completion of CCA_1, then Node_A proceeds to conduct CCA_2, upon finding the channel to be idle, Node_A is set to transmit a data packet and waits for an acknowledgement signal in the network (lines 16 to 17). CCA_2 expires after completion of time slot $t_{2}$ (line 18). The algorithm declares the channel status as busy due to the failure of CCA_2 for the two scenarios considered. Furthermore, the algorithm incorporates an additional CCA to address the busy channel condition as mentioned below.

Case 1. The performance of an additional CCA (CCA_3) is described based on the first condition (line 21). After the end of time slot $t_{\text {backoff } 2 \text {, Node_A completes CCA_1 and }}$ CCA_2 at time slots $t_{1}$ and $t_{2}$, respectively. Alternatively, Node_B (node contending for channel access in the network) completes its CCA_1 at time slot $t_{2}$ (same time slot utilized by Node_A for conducting CCA_2). Here, at time slot $t_{2}$, CCA_2 employed by Node_A fails, since Node_B is also contending for channel access. To overcome the failure of CCA_2 in scenario 1, Node_A is set to perform an additional CCA (CCA_3) at time slot $t_{3}$ (line 22) for successful transmission of the data packet. 


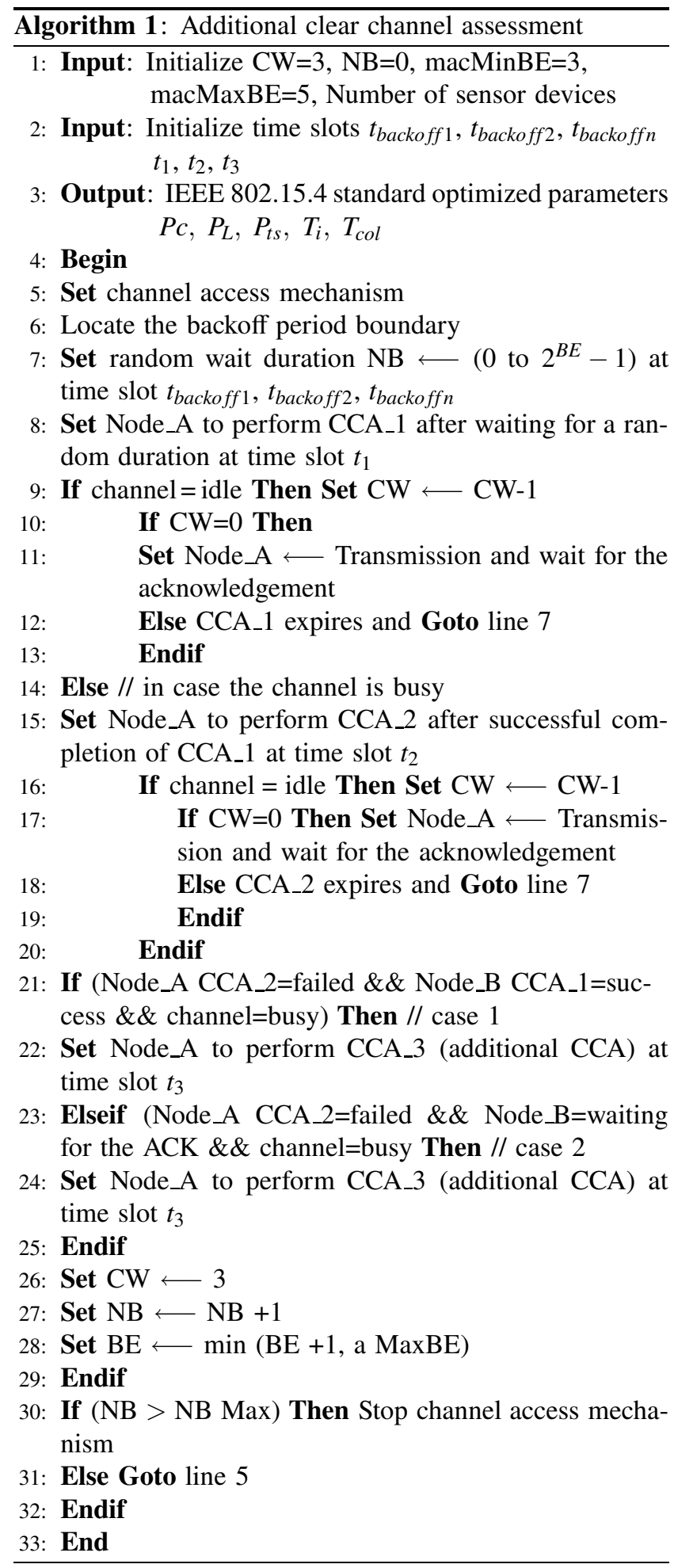

Case 2. The performance of an additional CCA (CCA_3) is described based on the second condition (line 23). After the end of time slot $t_{\text {backoff } 2}$, Node_A completes CCA_1 and CCA_2 at time slots $t_{1}$ and $t_{2}$, respectively. Alternatively, Node_B (node contending for channel access in the network) complete its CCA_2 at time slot $t_{2}$ (same time slot as utilized by Node_A for conducting CCA_1). Next, Node_B waits for an acknowledgement signal from the PAN coor- dinator node in the network. Here, at time slot $t_{2}$, CCA_2 employed by the Node_A fails, since Node_B is also contending for channel access. In order to overcome the failure of CCA_2 in scenario 2, Node_A is set to perform an additional CCA (CCA_3) at time slot $t_{3}$ (line 24) for successful transmission of the data packet.

Inputs $\mathrm{CW}, \mathrm{NB}, \mathrm{BE}$ are updated (lines 26-28) after the completion of the additional CCA. Finally, the algorithm checks if the maximum number of backoffs has been exceeded (line 30). If the condition is satisfied, the algorithm stops channel sensing, else it repeats line 31 and performs an additional CCA at time slot $t_{3}$ instead of waiting for a random backoff period and thus improves the overall efficiency of the network.

\section{Analytical Model}

A Markov chain model is developed for the proposed additional CCA in order to tackle the two scenarios mentioned in Section 3. The total number of nodes equals $N_{T}$. They are connected forming a star topology network with a PAN coordinator node. The stochastic processes for conducting the additional CCA are modeled using a Markov chain model illustrated in Fig. 5. We consider $s(t)$ as the number of backoff stages, $m(t)$ as the contention window, $n(t)$ as the counter of backoff stages, $t_{S C}(t)$ as the successful transmit cycle and collision cycle, respectively, where $s(t) \in\{0,2\}, m(t) \in\left\{-1,0, \ldots, W_{j}\right\}, n(t) \in\{0,1,2,3\}$ and $t_{S C}(t) \in\left\{0,1,2, \ldots, L_{f}\right\}$.

$$
W_{j}=2^{\operatorname{amin} B E+i}, \quad 0 \leq i \leq 2 .
$$

Let

$$
S_{i, j, k, l}=\lim _{t \rightarrow \infty} P\left\{s(t)=i, m(t)=j, n(t)=k, t_{S C}(t)=1\right\},
$$

where

$$
i \in[0,2], j \in\left[-1, W_{j}\right], k \in[0,3], l \in\left[0, L_{f}\right] .
$$

State $S_{i, j, k, l}$ represents changes that occurred in the channel access mechanism during the additional CCA. States from $(i, j, k)$ to $(i, j-1, k)$ represent the number of backoff states. Therefore, during the backoff period, a number of sensor devices in the network are in idle or sleep modes. States from $(i, j, k)$ to $(i, j-1, k-2)$ represent the number of CCAs conducted. A number of sensor devices are at this stage during the busy state of a wireless channel. States from $(i, j, l)$ to $(i, j, l+1)$ represent a successful transmission of the packet and the probability of a collision. The number of sensor devices will be in a transmission cycle during the idle state of the wireless channel. Also, the probability of a collision represents the number of sensor devices that experienced collisions during transmission of the data packet or its acknowledgement. Let $x_{1}$ be the probability condition for CCA during the first channel access attempt, $x_{2}$ be the probability condition for conducting CCA during the second channel access attempt caused by the previous 


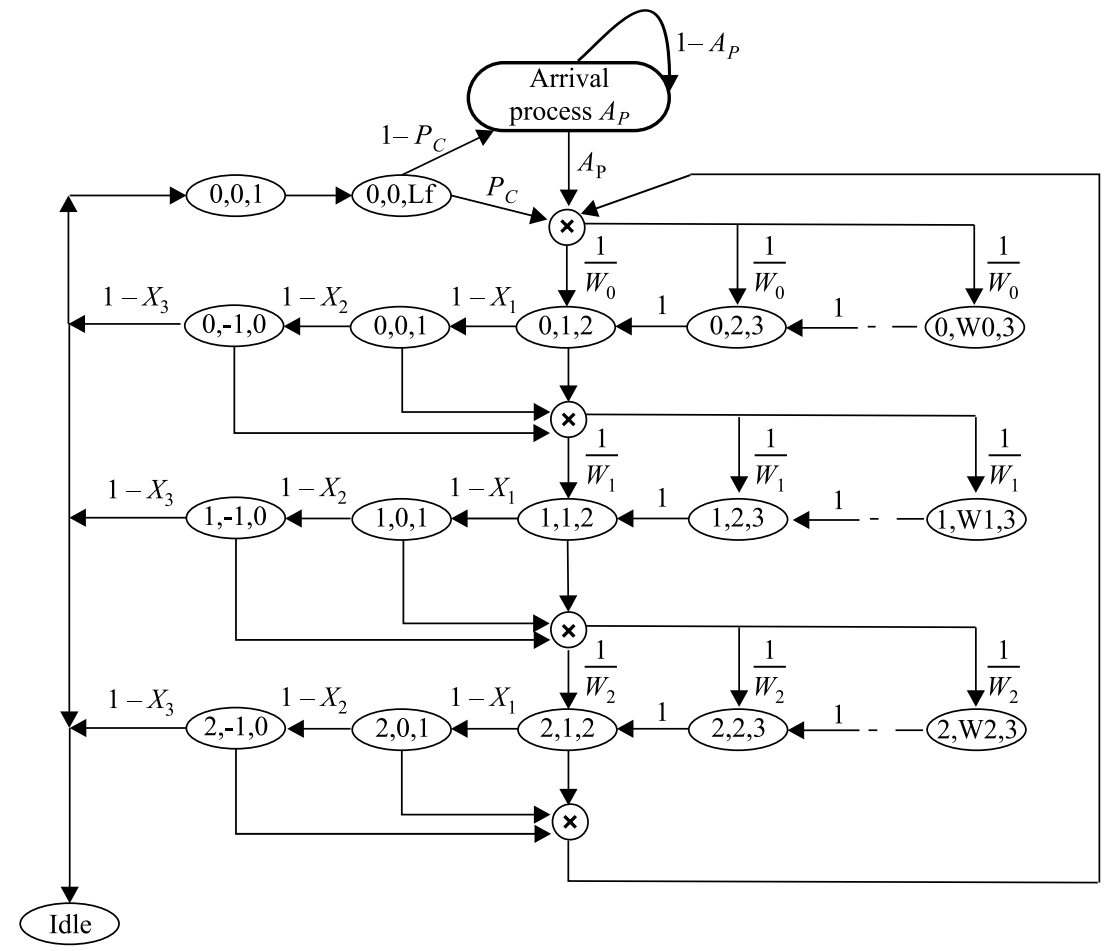

Fig. 5. Markov chain model for additional CCA.

idle channel condition, and $x_{3}$ be the probability condition for an additional CCA.

As depicted in Fig. 5, the state transition probabilities are:

$$
\begin{gathered}
P\{i, j-1, k \mid i, j, k\}=1, \quad j \in\left[-1, W_{j}\right], \\
P\{i, j-1, k-2 \mid i, j, k\}=\left(1-x_{1}\right)\left(1-x_{2}\right)\left(1-x_{3}\right), \\
j \in\left[-1, W_{j}\right], \quad k \in[0,3], \\
P\{i, j, l+1 \mid i, j, l\}=\left(1-x_{1}\right)\left(1-x_{2}\right)\left(1-x_{3}\right)\left(1-P_{C}\right), \\
l \in\left[0, L_{f}\right], \\
P\{i, j, l+1 \mid i, j, l\}=\left(1-x_{1}\right)\left(1-x_{2}\right)\left(1-x_{3}\right) P_{C}, \\
l \in\left[0, L_{f}\right] .
\end{gathered}
$$

Equation (3) represents the state transition with probability 1 , whenever there is a backoff counter decrement, while Eq. (4) represents the state transition after the additional CCA has been conducted. Equation (5) represents the state transition for a successful cycle and Eq. (6) represents the state transition for a collision cycle. Table 1 provides the list of symbols used in the analytical model.

\section{Performance Metrics}

In this section we define the various performance metrics of the additional CCA, such as probability of collision, packet loss rate, probability of data transmission, probability of a successful data transmission, channel utilization, channel collision time, channel idle time, reliability, delay, energy consumption of a node, and investigate their impact on the IEEE 802.15.4-based MAC protocol. While conducting the CCA, the node's state changes based on the traffic generated in the network. Channel sensing probability defines probability changes associated with a given node, i.e. the node may be in the idle, collision, busy and transmit state.

The channel sensing probability is:

$$
\emptyset=\frac{2}{W_{b}^{\max }+1+K 1+K 2},
$$

where

$$
\begin{gathered}
K 1=2 L_{f}\left(1-x_{1}\right)\left(1-x_{2}\right)\left(1-x_{3}\right), \\
K 2=2\left[\left(1-x_{1}\right)+\left(1-x_{1}\right)\left(1-x_{2}\right)\right],
\end{gathered}
$$

Whenever nodes contend for wireless channel access, there is a chance of packet collisions in the network. This metric provides details of all nodes which suffered from packet collisions in the network while accessing the channel. The nodes experience packet collisions due to simultaneous data packet transmissions performed by all nodes. Hence, collision probability always increases with the number of nodes in the network and is defined as the probability of all nodes being in the collision state:

$$
P_{C}=1-(1-\emptyset)^{N_{T}-1} .
$$


Table 1

List of symbols used

\begin{tabular}{|c|c|}
\hline Symbol & Description \\
\hline$P(i, j, k)$ & State transition probability \\
\hline$T_{m e a n}$ & Average time \\
\hline$T_{\text {busy }}$ & Total time during busy cycle \\
\hline$T_{b o f}$ & Total backoff time \\
\hline$T_{c c}$ & Total time for CCA conduction \\
\hline$T_{c o l}$ & Total time during collision cycle \\
\hline$T_{\text {suc }}$ & Total time during success cycle \\
\hline$T_{r c v}$ & Total time during receive cycle \\
\hline$T_{i}$ & Time during channel in idle state \\
\hline$T_{a}$ & Turnaround time during receive cycle \\
\hline$T$ & Total time \\
\hline$n_{F}^{\max }$ & Maximum value for frame retries \\
\hline$W_{b}^{\max }$ & Maximum value for random back off time \\
\hline$N_{T}$ & Total nodes in a network \\
\hline$N_{\text {act }}$ & Total active nodes in a network \\
\hline$N_{C C A}$ & Total nodes conducting CCA \\
\hline$R$ & Reliability of a network \\
\hline$\emptyset$ & Sensing probability of channel \\
\hline$\emptyset_{11}$ & Sensing probability of channel in state $S_{11}$ \\
\hline$P_{c}$ & Probability of collision \\
\hline$P_{t d}$ & Probability of data transmission \\
\hline$P_{t s}$ & Probability of successful data transmission \\
\hline$C_{u t}$ & Channel utilization \\
\hline$\pi_{\text {suc }}$ & Transition for success cycle \\
\hline$\pi_{r c v}$ & Transition for receive cycle \\
\hline$\pi_{\text {busy }}$ & Transition for busy cycle \\
\hline$\pi_{c o l}$ & Transition for collision cycle \\
\hline$P_{t}$ & Power consumed during transmission mode \\
\hline$P_{r}$ & Power consumed during receive mode \\
\hline$P_{c c}$ & Power consumed during CCA conduction \\
\hline$P_{i}$ & Power consumed during idle mode \\
\hline$P_{L}$ & Packet loss rate \\
\hline$L_{f}$ & Length of the frame \\
\hline$E_{T c c}$ & Total energy consumed for CCA computation \\
\hline$E_{T b}$ & Total energy consumed for backoff state \\
\hline$E_{T}$ & Total energy for all states \\
\hline$E_{t}$ & Total energy consumed for transmitting \\
\hline$E_{r}$ & Total energy consumed for receiving \\
\hline$X$ & Success state \\
\hline Y & Busy state \\
\hline$Z$ & Collision state \\
\hline
\end{tabular}

As nodes encounter repeated collisions in the network while accessing the wireless channel, a parameter known as packet loss rate may be defined. Efficiency of the network is degraded if the packet loss rate is high. Therefore, the packet loss rate determines the number of packets lost in the network and depends on the number of nodes in the network:

$$
P_{L}=\left[1-(1-\emptyset)^{N_{T}-1}\right]^{n_{F}^{\max }+1} .
$$

The probability of finding any single node transmitting a data packet after the performance of the additional CCA is equal to the probability of data transmission. This metric determines the number of nodes involved in data transmission after successful completion of the additional CCA, without acknowledgment from the receiver. In other words, this metric does not provide any information about the packets lost due to collisions and about the acknowledgement received from the other nodes in the network. Therefore, the probability of data transmission is:

$$
P_{t d}=1-(1-\emptyset)^{N_{T}}
$$

The probability of a successful data transmission is expressed as:

$$
P_{t s}=\frac{N_{T} \emptyset(1-\emptyset)^{N_{T}-1}}{P_{t d}} .
$$

This metric is defined as the probability of a successful transmission of data packets without any collisions in the network. It is determined based on the acknowledgement received by the other nodes in the network.

Effective channel utilization is the most important metric measured in order to determine network efficiency. It is possible to achieve a maximum channel utilization rate in a network if all nodes spend more time transmitting or receiving data packets without any collisions and spend less time on other activities, such as CCA, and in busy states. Let $N_{\text {act }}$ be the number of active sensor nodes in a network, contending for channel access. Then, effective channel utilization $C_{u t}$ is:

$$
C_{u t}=N_{a c t} L_{f} \emptyset(1-\emptyset)^{N_{T}-1}\left(1-x_{1}\right)\left(1-x_{2}\right)\left(1-x_{3}\right) .
$$

In WSNs, it is always better to keep the wireless channel busy with performing any activities, such as data packet transmission or reception between the nodes. All nodes should be busy, either conducting CCA or busy with the backoff states. Therefore, performance of the network is degraded if a channel is in the idle state. Channel idle time is defined as the wireless channel not performing any activities in the network. The idle time parameter should be as low as possible, and it is always a complementary phase in the process of conducting a clear channel assessment (CCA) during the first channel access attempt. It is given by:

$$
T_{i}=1-x_{1}
$$

In WSNs, if there are more nodes contending for access to a given channel, then the probability of a collision will always be high. In order to enhance the channel utilization rate of a network, we need to ensure that the total time spent by all nodes dealing with packet collisions should be as low as possible. The channel collision time 
is defined as the total time spent by all nodes in the collision state:

$$
T_{c o l}=x_{1}-N_{a c t} L_{f} \emptyset(1-\emptyset)^{N_{T}-1}\left(1-x_{1}\right)\left(1-x_{2}\right)\left(1-x_{3}\right)
$$

In CCA, the reliability of a network plays a major role in determining network efficiency. Reliability of the additional CCA is determined based upon state transitions occurring in the network while accessing the wireless channel. Whenever a node performs CCA, it enters into three possible states, such as success state, busy state, and collision state. In the success state, the node delivers data packets to the destination node, in the busy state, the node is waiting for the channel, and in the collision state, the node experiences packet collisions. This metric is defined as the probability of a node being in the success state without any packet collisions:

$$
R=\frac{1}{1+\frac{[1-Y\} Y^{m+1}}{\left[1-Y^{m+1}\right] X}+\frac{Z^{n+1}}{\left((1-Y)^{n+1}\right)-z^{n+1}}}
$$

In WSNs, all nodes are battery powered and energy consumption of a node plays a major role whenever an additional CCA is performed in the IEEE 802.15.4-based MAC protocol. Hence, energy consumption of a node is computed based on the data packet transmitted or received by that node, as well as based on the node's state change (i.e. collision or busy state):

$$
E_{T}=E_{t}+E_{r}+E_{T b}+E_{T c c}
$$

$$
\begin{aligned}
E_{T}=\left(P_{t}+P_{r}\right) L_{f} & (1-\emptyset)^{N_{T}-1}\left(1-x_{1}\right)\left(1-x_{2}\right) \emptyset_{11} \\
& +P_{i} \frac{W_{b}^{\max }-1}{2} \emptyset_{11}+P_{c c}\left[2-x_{1}\right] \emptyset_{11}
\end{aligned}
$$

The average delay is defined as the total time needed to complete the successful transmission of the data packet by a node. It determines the overall delay experienced by the node whenever an additional CCA is performed. Therefore, computation of the overall delay of a node is based on its state transitions occurring in the network. Further, we express the overall delay based on the time spent by the node on completing the successful transmission or on receiving a data packet, time spent in the busy state, and time spent in the collision state. The overall delay is:

$$
T=T_{s u c}+T_{r c v}+T_{b u s y}+T_{c o l}
$$

The mean delay is:

$$
\begin{aligned}
T_{\text {mean }}=[1+ & \left.\frac{\pi_{c o l}}{\pi_{s u c}}+\frac{\pi_{r c v}}{\pi_{s u c}}+\frac{\pi_{b u s y}}{\pi_{s u c}}\right] T_{b o f}+\frac{\pi_{b u s y}}{\pi_{s u c}} T_{c c} \\
+ & {\left[1+\frac{\pi_{c o l}}{\pi_{s u c}}+\frac{\pi_{r c v}}{\pi_{s u c}}\right]\left[3+L_{f}\right]+\frac{\pi_{r c v}}{\pi_{s u c}} T_{a} . }
\end{aligned}
$$

\section{Performance Evaluation}

The performance of the additional CCA for the analytical model developed in Section 5 is evaluated using Matlab software. The impact of additional CCA on some of the performance metrics in the IEEE 802.15.4 based MAC protocol is investigated, and the performance metrics are analyzed. Table 2 provides the simulation parameters used in the model.

Table 2

Simulation parameters

\begin{tabular}{|c|c|}
\hline Parameter & Value \\
\hline \hline Band & $2.4 \mathrm{GHz}$ \\
\hline Channel bandwidth & $250 \mathrm{kbps}$ \\
\hline Channel number & 11 \\
\hline Unit backoff period & 20 symbols \\
\hline Number of nodes & $5-500$ \\
\hline Turnaround time $\left(T_{a}\right)$ & 12 symbols \\
\hline Transmission power consumed $\left(P_{t}\right)$ & $32 \mathrm{~mW}$ \\
\hline Receiving power consumed $\left(P_{r}\right)$ & $38 \mathrm{~mW}$ \\
\hline CCA power consumed $\left(P_{c c}\right)$ & $38 \mathrm{~mW}$ \\
\hline Idle power consumed $\left(P_{i}\right)$ & $0.8 \mathrm{~mW}$ \\
\hline macMaxCSMABackoffs & 5 \\
\hline macMaxFrameRetries & 4 \\
\hline macMinBE & 3 \\
\hline macMaxBE & 5 \\
\hline Frame length $\left(L_{f}\right)$ & 12 time slots \\
\hline
\end{tabular}

As depicted in Fig. 6, it is clear that the probability of a collision increases with the number of devices in the network. The probability of a collision for the IEEE 802.15.4 standard is $15.27 \%$ at $N=5,67.58 \%$ at $N=100$, and $98.06 \%$ at $N=500$, respectively. Similarly, the probability of a collision for the AS 802.15.4 MAC protocol is $12.20 \%$ at $N=5,63.54 \%$ at $N=100$, and $96.06 \%$ at $N=500$, respectively. Finally, the probability of a colli-

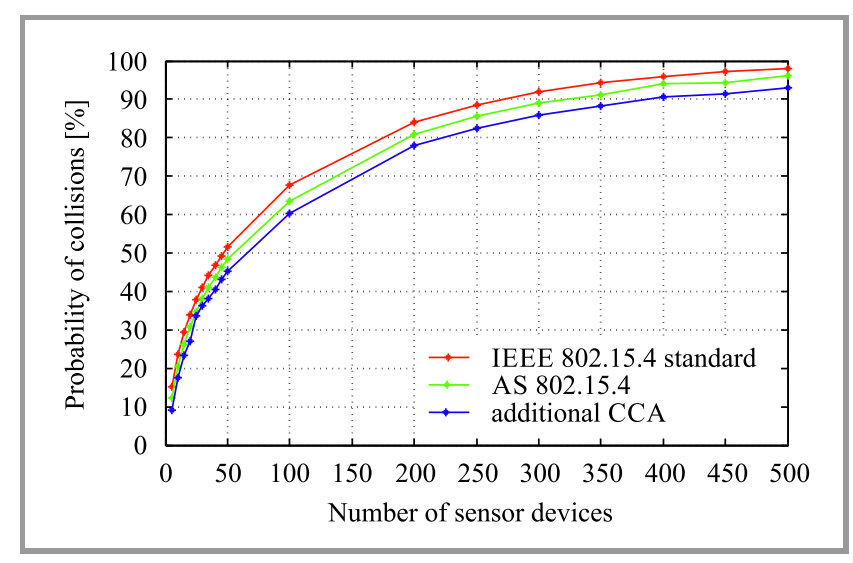

Fig. 6. Probability of collision versus network size. 
sion for the additional CCA conducted is $9.16 \%$ at $N=5$, $60.32 \%$ at $N=100$, and $93.12 \%$ at $N=500$, respectively. The probability of a collision is reduced with an additional CCA in comparison with IEEE 802.15.4 and AS 802.15.4 MAC.

Figure 7 presents an evaluation of the packet loss rate parameter. When compared to the IEEE 802.15.4 and AS 802.15.4, the packet loss rate is reduced whenever an additional CCA is performed. It is evident from Fig. 7 that the packet loss rate for IEEE 802.15 .4 is $0.05 \%$ at $N=5$, $20.85 \%$ at $N=100$, and $92.47 \%$ at $N=500$, respectively. Similarly, the packet loss rate for the AS 802.15 .4 is $0.03 \%$ at $N=5,17.81 \%$ at $N=100$, and $89.45 \%$ at $N=500$, respectively. Finally, the packet loss rate for the additional CCA conducted is $0.01 \%$ at $\mathrm{N}=5,13.75 \%(N=100)$, and $86.53 \%(N=500)$, respectively.

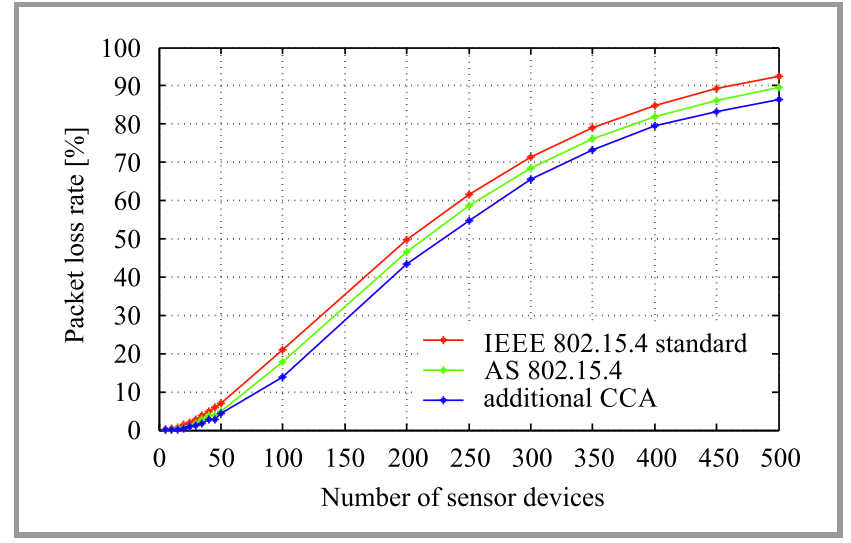

Fig. 7. Packet loss rate versus network size.

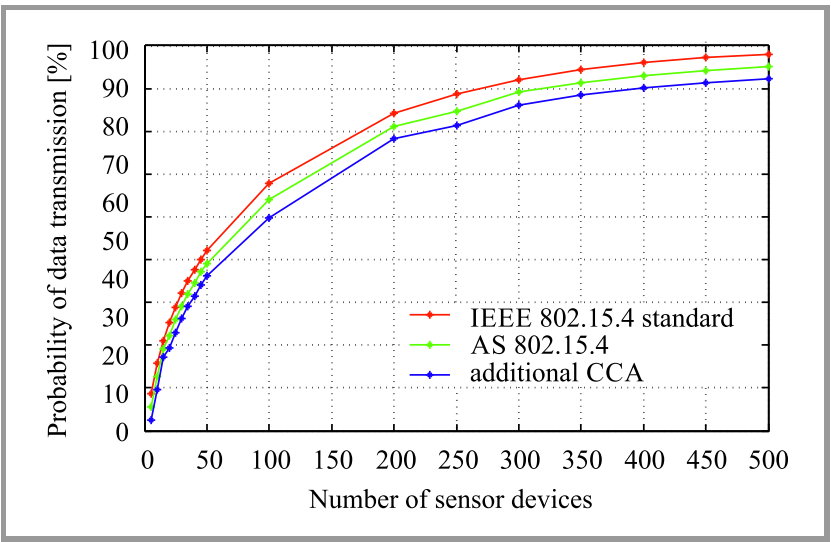

Fig. 8. Probability of data transmission versus network size.

Figure 8 shows an evaluation of data transmission probability. When compared to the existing standards, the probability of data transmission is reduced with an additional CCA. It is observed from Fig. 8 that the probability of data transmission for the IEEE 802.15.4 standard is $18.71 \%$ at $N=5,67.94 \%$ at $N=100$, and $98.08 \%(N=500)$, respectively. Similarly, the probability of data transmission for the AS 802.15.4 MAC protocol is $15.63 \%$ at $N=5$, $63.91 \%(N=100)$, and $95.07 \%(N=500)$, respectively.
Finally, the probability of data transmission for the additional CCA is $12.56 \%$ at $N=5,59.78 \%(N=100)$, and $92.32 \%(N=500)$, respectively.

Figure 9 presents an evaluation of the probability of successful data transmission. When compared to the IEEE 802.15.4 and AS 802.15.4 MAC protocol, the probability of successful data transmission is significantly enhanced whenever an additional CCA is conducted. The probability of successful data transmission for the additional CCA conducted is $97.87 \%$ at $N=5,59.17 \%$ at $N=100$, and $13.54 \%$ at $N=500$, respectively.

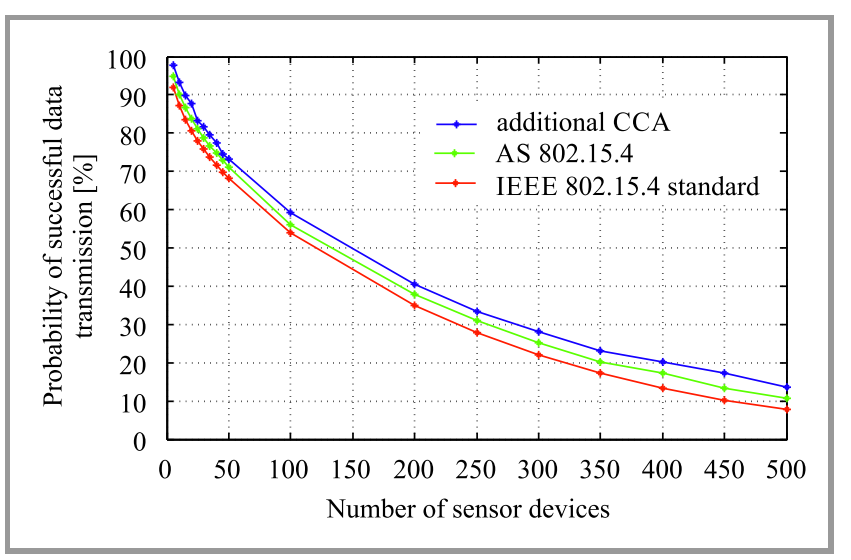

Fig. 9. Probability of successful data transmission versus network size.

As shown in Fig. 10, effective channel utilization is high initially, because of the lower number of nodes contending for channel access. It decreases later on due to the additional nodes being present in the network. It is observed from Fig. 10 that channel utilization with the additional CCA is $69.79 \%(N=5), 43.48 \%(N=100)$, and $3.98 \%$ at $(N=500)$, respectively. The channel utilization metric is degraded as the additional CCA is introduced.

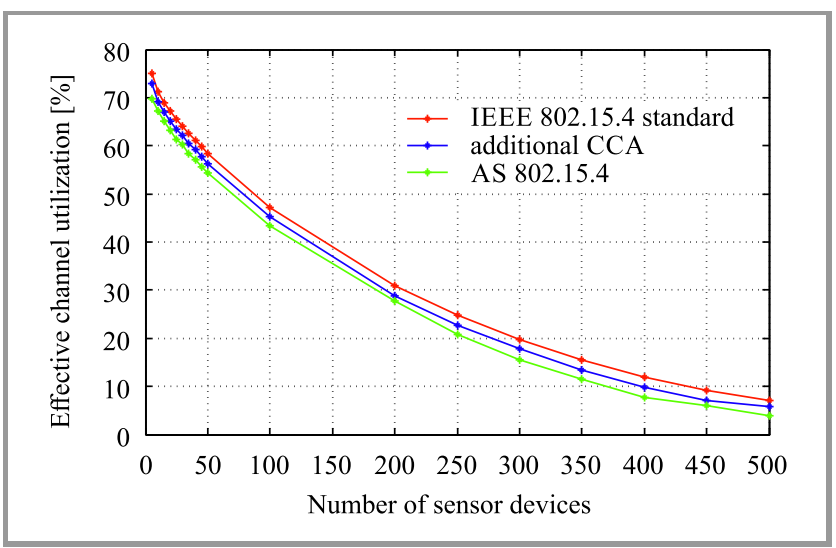

Fig. 10. Effective channel utilization versus network size.

Figure 11 clearly shows that the channel idle time is high initially, because the probability of a channel being in the idle state is higher when compared to later stages, due to the additional nodes in the network. Channel idle time for the additional CCA conducted is $37.99 \%(N=5), 19.91 \%$ 
$(N=100)$, and $17.74 \%(N=500)$, respectively. Channel idle time is higher whenever an additional CCA is conducted in comparison to existing standards.

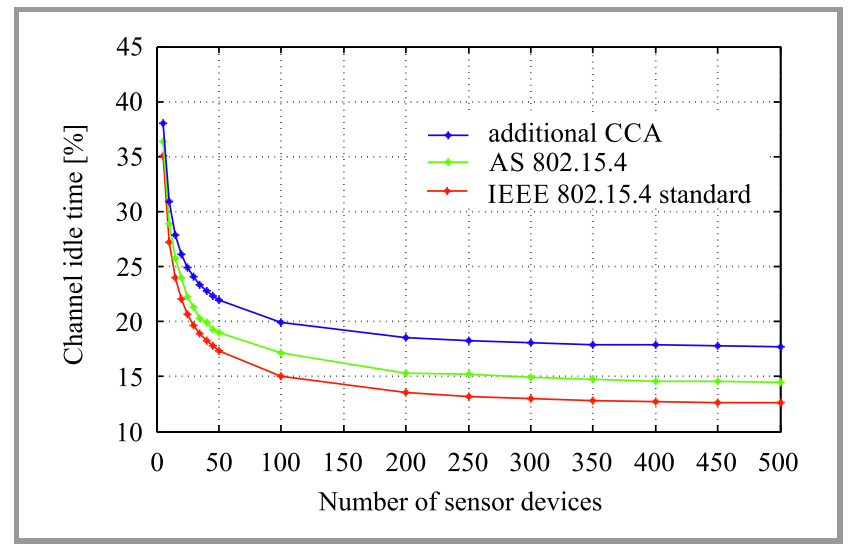

Fig. 11. Channel idle time versus network size.

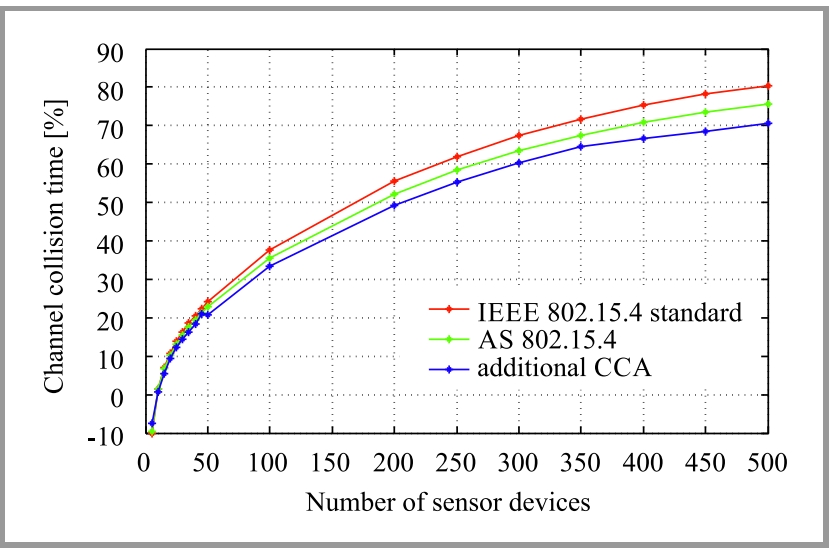

Fig. 12. Channel collision time versus network size.

Channel collision time always increases along with the number of nodes in the network (Fig. 12). Channel collision time for the additional CCA conducted is $-7.62 \%$ at $N=5,33.48 \%$ at $N=100$, and $70.71 \%$ at $N=500$, respectively. Channel collision time is reduced whenever an additional CCA is performed.

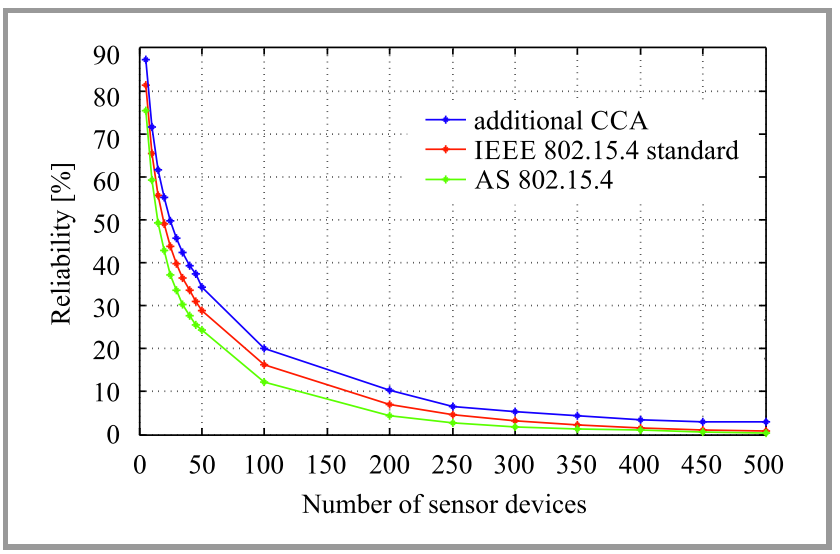

Fig. 13. Reliability versus network size.
As depicted in Fig. 13, reliability of the network is reduced with an increase in the number of nodes. Reliability for the additional CCA is $87.32 \%$ at $N=5,20.13 \%$ at $N=100$, and $2.85 \%$ at $N=500$, respectively. The reliability of a network is enhanced with the additional CCA, compared to the IEEE 802.15.4 standard and the AS 802.15.4 MAC protocol.

Energy consumption of a node in the case of the IEEE 802.15 .4 standard is $4.1757 \mathrm{~J}$ at $N=5,0.5189 \mathrm{~J}$ at $N=100$, and $0.3684 \mathrm{~J}$ at $N=500$ respectively (Fig. 14). Similarly, the energy consumption of a node for the AS 802.15.4 MAC protocol is $4.5216 \mathrm{~J}$ at $N=5,0.6456 \mathrm{~J}$ at $N=100$, and $0.4566 \mathrm{~J}$ at $N=500$, respectively. Energy consumption of a node for the additional CCA conducted is $4.8122 \mathrm{~J}$ at $N=5,0.8721 \mathrm{~J}$ at $N=100$, and $0.6751 \mathrm{~J}$ at $N=500$, respectively. Therefore, energy consumption of the node increases with the additional CCA.

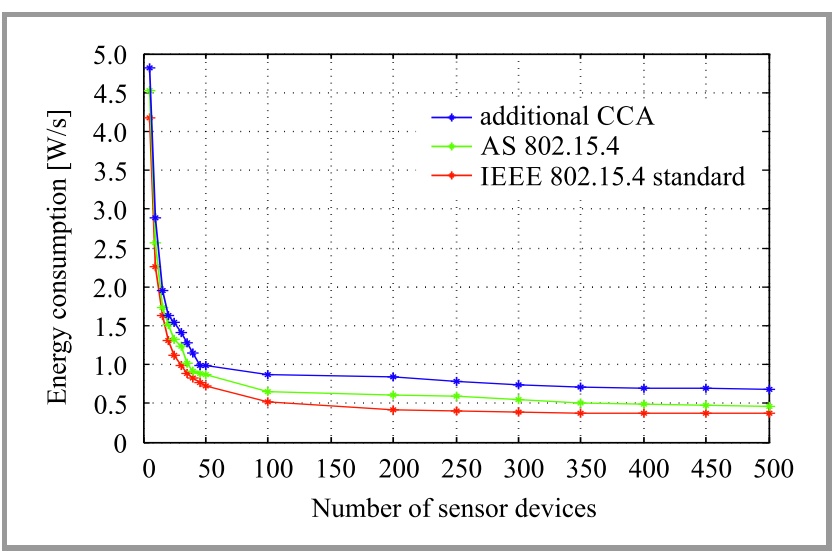

Fig. 14. Energy consumption versus network size.

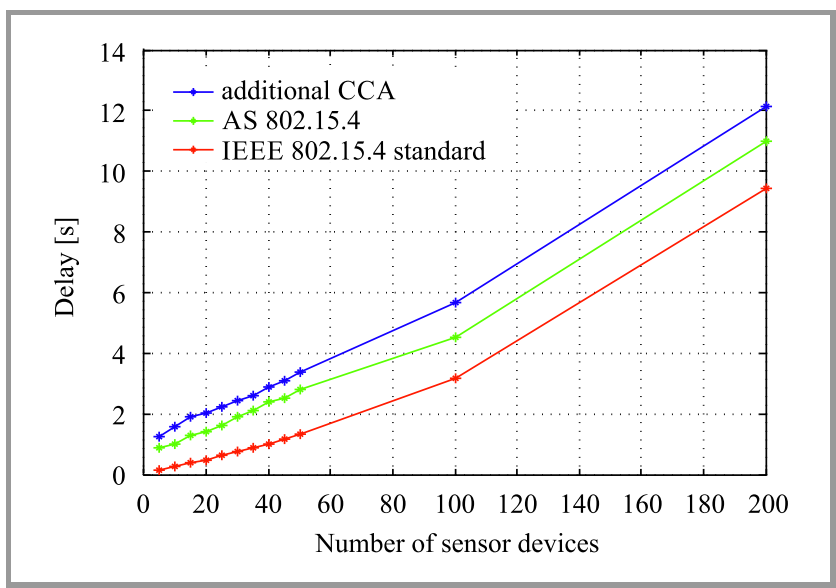

Fig. 15. Delay versus network size.

As shown in Fig. 15, the average delay achieved by a node for the IEEE 802.15.4 standard is $0.1332 \mathrm{~s}$ at $N=5$, and $3.1559 \mathrm{~s}$ at $N=100$, respectively. Similarly, delay for the AS 802.15.4 MAC protocol is $0.8687 \mathrm{~s}(N=5)$, and $4.5403 \mathrm{~s}(N=100)$, respectively. Delay for the additional CCA conducted is $1.2578 \mathrm{~s}$ at $N=5$, and $5.6534 \mathrm{~s}$ 
at $N=100$. One may notice that higher delays are caused by conducting the additional CCA.

Table 3

Comparative analysis of existing and proposed standards

\begin{tabular}{|c|c|c|c|}
\hline $\begin{array}{c}\text { Parameter average } \\
\text { (relative) }\end{array}$ & $\begin{array}{c}\text { IEEE } \\
802.15 .4 \\
\text { standard }\end{array}$ & $\begin{array}{c}\text { AS } \\
802.15 .4 \\
\text { standard }\end{array}$ & $\begin{array}{c}\text { Additional } \\
\text { CCA }\end{array}$ \\
\hline \hline $\begin{array}{c}\text { Probability of } \\
\text { collision }\end{array}$ & $60.56 \%$ & $57.59 \%$ & $54.66 \%$ \\
\hline Packet loss rate & $32.134 \%$ & $30.25 \%$ & $28.58 \%$ \\
\hline $\begin{array}{c}\text { Probability of data } \\
\text { transmission }\end{array}$ & $61.41 \%$ & $58.33 \%$ & $55.36 \%$ \\
\hline $\begin{array}{c}\text { Probability of } \\
\text { successful data } \\
\text { transmission }\end{array}$ & $53.77 \%$ & $56.80 \%$ & $59.66 \%$ \\
\hline $\begin{array}{c}\text { Effective channel } \\
\text { utilization }\end{array}$ & $45.55 \%$ & $41.60 \%$ & $43.57 \%$ \\
\hline Channel idle time & $18.14 \%$ & $19.88 \%$ & $22.69 \%$ \\
\hline Channel collision time & $36.29 \%$ & $34.17 \%$ & $32.19 \%$ \\
\hline Reliability & $27.87 \%$ & $23.87 \%$ & $32.25 \%$ \\
\hline Energy consumption & $0.9933 \mathrm{~J}$ & $1.1605 \mathrm{~J}$ & $1.3691 \mathrm{~J}$ \\
\hline Delay & $1.6322 \mathrm{~s}$ & $2.7906 \mathrm{~s}$ & $3.4256 \mathrm{~s}$ \\
\hline
\end{tabular}

Table 3 presents a comparative analysis of all performance metrics investigated for the IEEE 802.15.4 standard, the AS 802.15.4 MAC protocol, and the additional CCA conducted. The average values of all performance metrics are considered. Furthermore, simulation results show a difference between the IEEE 802.15.4 standard, the AS 802.15.4 MAC protocol, and the additional CCA. From Table 3, it is evident that some of the performance metrics are improved in the IEEE 802.15.4 standard-based MAC protocol by conducting an additional CCA.

\section{Conclusion}

In this paper, we have modeled and analyzed an additional CCA incorporated in the IEEE 802.15.4-based MAC protocol, in a star topology-enabled WSNs. The proposed method addresses the busy channel status encountered in two scenarios considered during the channel access mechanism. In both scenarios, the proposed method relies on an additional CCA in order to enable collision-free data transmission to be performed by all nodes in the network. Initially, a Markov chain model and a set of mathematical equations were developed for the proposed method. Later, we investigated the impact of the additional CCA on certain performance metrics in the IEEE 802.15.4-based MAC protocol. It could be observed that some of the performance metrics regarding the IEEE 802.15.4 standard improved with the introduction of the additional CCA. Simulation results show that there is a significant improvement in performance metrics, such as probability of collision, packet loss rate, probability of successful data transmission, channel idle time, channel collision time, and the network reliability.

\section{References}

[1] P. Park, P. Di Marco, C. Fischione, and K. H. Johansson, "Modeling and optimization of the IEEE 802.15.4 protocol for reliable and timely communications", IEEE Trans. on Parall. and Distrib. Syst., vol. 24, no. 3, pp. 550-564, 2013

(DOI: 10.1109/TPDS.2012.159).

[2] M. Khanafer, M. Guennoun, and H. T. Mouftah, "A survey of beacon-enabled IEEE 802.15.4 MAC protocols in wireless sensor networks", IEEE Commun. Surv. \& Tutor., vol. 16, no. 2, pp. 856-876, 2014 (DOI: 10.1109/SURV.2013.112613.00094).

[3] M. P. R. S. Kiran and P. Rajalakshmi, "Performance analysis of CSMA/CA and PCA for time critical industrial IoT applications", IEEE Trans. on Indust. Inform., vol. 14, no. 5, pp. 2281-2292, 2018 (DOI: 10.1109/TII.2018.2802497).

[4] D. De Guglielmo, F. Restuccia, G. Anastasi, M. Conti, and S. K. Das, "Accurate and efficient modeling of 802.15.4 unslotted CSMA/CA through event chains computation", IEEE Trans. on Mob. Comput., vol. 15 , no. 12 , pp. 2954-2968, 2016 (DOI: 10.1109/TMC.2016.2528248).

[5] B. Bandyopadhyay S. J. Ahmed, D. Das, and A. Chatterjee, "AS 802.15.4: A modified IEEE 802.15.4 standard for more reliable communication and utilization of inactive period using optimized sleep period", in Proc. of Ann. IEEE India Conf. INDICON 2014, Pune, India, 2014 (DOI: 10.1109/INDICON.2014.7030421).

[6] X. Cao, J. Chen, Y. Cheng, X. S. Shen, and Y. Sun, "Analytical MAC model for IEEE 802.15.4 enabled wireless networks with periodic traffic", IEEE Trans. on Wirel. Commun., vol. 14, no. 10, pp. 5261-5273, 2015 (DOI: 10.1109/TWC.2015.2435006).

[7] B.-H. Lee, R.-L. Lai, H.-K. Wu, and C.-M. Wong, "Study on additional carrier sensing for IEEE 802.15.4 wireless sensor networks", Sensors, vol. 10, no. 7, pp. 6275-6289, 2010 (DOI: 10.3390/s100706275).

[8] M. Guennoun, M. Khanafer, and H. T. Mouftah, "Modeling of variable clear channel assessment MAC protocol for wireless sensor networks", Сomp. Commun., vol. 59, pp. 67-83, 2015 (DOI: 10.1016/j.comcom.2015.01.008).

[9] B. Khandish, E. Lee, H. Park, and J.-B. Suk, "An efficient backoff scheme in wireless sensor networks", in Proc. 10th Int. Conf. on Ubiquit. and Future Netw. ICUFN 2018, Prague, Czech Republic, 2018, pp. 332-337 (DOI: 10.1109/ICUFN.2018.8437013).

[10] J. Zhu, Z. Tao, and C. Lv, "Performance evaluation of IEEE 802.15.4 CSMA/CA scheme adopting a modified LIB model", Wirel. Pers. Commun., vol. 65, no. 1, pp. 25-51, 2012 (DOI: 10.1007/s11277-011-0226-6).

[11] N. R. Patel and S. Kumar, "Enhanced clear channel assessment for slotted CSMA/CA in IEEE 802.15.4”, Wirel. Pers. Commun., vol. 95, pp. 4063-4081 (DOI: 10.1007/s11277-017-4042-5).

[12] S. Chen, T. Sun, J. Yuan, and X. Geng, "Performance analysis of IEEE 802.15.4e time slotted channel hopping for low-rate wireless networks, KSII Trans. on Internet and Inform. Syst., vol. 7, no. 1, pp. 1-21, 2013 (DOI: 10.3837/tiis.2013.01.001).

[13] P. Di Marco, C. Fischione, F. Santucci, and K. H. Johansson, "Modeling IEEE 802.15.4 networks over fading channels", IEEE Trans. on Wirel. Commun., vol. 13, no. 10, pp. 5366-5381, 2014 (DOI: 10.1109/TWC.2014.2349499).

[14] S. R. Pattanaik, P. K. Sahoo, and S.-L. Wu, "Performance analysis of modified IEEE 802.15.4e MAC for wireless sensor networks", in Proc. of 14th ACM Symp. on Perform. Eval. of Wirel. Ad Hoc, Sensor, \& Ubiquit. Netw. WASUN'17, Miami, FL, USA, 2017, pp. 25-31 (DOI: 10.1145/3134829.3134833).

[15] K. J. Son, S. H. Hong, and S.-P. Moon, "Segmentized clear channel assessment for IEEE 802.15.4 networks", Sensors, vol. 16, no. 6, pp. 1-16, 2016 (DOI: 10.3390/s16060815). 
[16] Y. Zhang et al., "A blind adaptive tuning algorithm for reliable and energy-efficient communication in IEEE 802.15.4 networks", IEEE Trans. on Veh. Technol., vol. 66, no. 9, pp. 8605-8609, 2017 (DOI: 10.1109/TVT.2017.2686453).

[17] P. K. Sahoo, S. R. Pattanaik, and S.-L. Wu, "A reliable data transmission model for IEEE 802.15.4e enabled wireless sensor network under WiFi interference", Sensors, vol. 17, no. 6, pp. 1-28, 2017 (DOI: 10.3390/s17061320).

[18] L. Zhao, G.-w. Bai, H. Shen, Z.-m. Tang, "Priority-based IEEE 802.15.4 CSMA/CA mechanism for WSNs", The J. of China Univer. of Posts and Telecommun., vol. 20, no. 1, pp. 47-53, 2013 (DOI: 10.1016/S1005-8885(13)60006-0).

[19] I. Bouazzi, J. Bhar, and M. Atri, "Analysis of the IEEE 802.15.4 MAC parameters to achieve lower packet loss rates", Procedia Comp. Sci., vol. 73, pp. 443-451, 2015 (DOI: 10.1016/j.procs.2015.12.021).

[20] F. Ullah, A. H. Abdullah, G. Abdul-Salaam, and M. M. Arshad, "CDASA-CSMA/CA: Contention differentiated adaptive slot allocation CSMA/CA for heterogeneous data in wireless body area networks", KSII Trans. on Internet and Inform. Syst., vol. 11, no. 12 , pp. $5835-5854,2017$ (DOI: 10.3837/tiis.2017.12.009).

[21] S. Moulik, S. Misra, and D. Das, "AT-MAC: Adaptive MACframe payload tuning for reliable communication in wireless body area networks", IEEE Trans. on Mob. Comput., vol. 16, no. 6, pp. 1516-1529, 2017 (DOI: 10.1109/TMC.2016.2598166).

[22] H. Rasouli, Y. S. Kavian, and H. F. Rashvand, "ADCA: Adaptive duty cycle algorithm for energy efficient IEEE 802.15.4 beaconenabled wireless sensor networks", IEEE Sensors J., vol. 14, no. 11, pp. 3893-3902, 2014 (DOI: 10.1109/JSEN.2014.2353574).

[23] K. J. Son, H. Cho, S. H. Hong, S.-P. Moon, and T. G. Chang, "New enhanced clear channel assessment method for IEEE 802.15.4 network", in Proc. Int. SoC Design Conf. ISOCC 2015, Gyungju, South Korea, 2015, pp. 251-252 (DOI: 10.1109/ISOCC.2015.7401742).

[24] Z. Dahham, A. Sali, B. M. Ali, and M. S. Jahan "An efficient CSMA/CA algorithm for IEEE 802.15.4 wireless sensor networks", in Proc. of IEEE Int. Symp. on Telecommun. Technol. ISTT 2012, Kuala Lumpur, Malaysia, 2012, pp. 118-123, 2012 (DOI: 10.1109/ISTT.2012.6481575)

[25] H. Lee et al., "An efficient slotted CSMA/CA algorithm for the IEEE 802.15.4 LR-WPAN", in Proc. of the Int. Conf. on Inform. Network. ICOIN 2011, Barcelona, Spain, 2011, pp. 488-493 (DOI: 10.1109/ICOIN.2011.5723164).

[26] M. Khanafer, M. Guennoun, and H. T. Mouftah, "Priority-based CCA periods for efficient and reliable communications in wireless sensor networks", Wirel. Sensor Netw., vol. 4, pp. 45-51, 2012 (DOI: 10.4236/wsn.2012.42007).

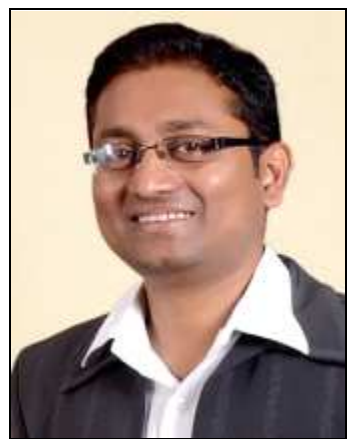

Manjunath G. Asuti is an Assistant Professor at the School of Electronics and Communication Engineering, REVA University, Bangalore, India. $\mathrm{He}$ received a B.E. in Electronics and Communication Engineering and an M.Tech. in VLSI and Embedded systems from Visvesvaraya Technological University, Belgaum, and is presently pursuing a Ph.D. at REVA University, Bangalore. He has 14 years of teaching experience. His areas of interest include wireless sensor networks, VLSI, DSP, embedded systems, and communication systems.

(iD) https://orcid.org/0000-0001-6577-7396

E-mail: manjunathgasuti@reva.edu.in

School of Electronics and Communication Engineering REVA University

Bengaluru, India

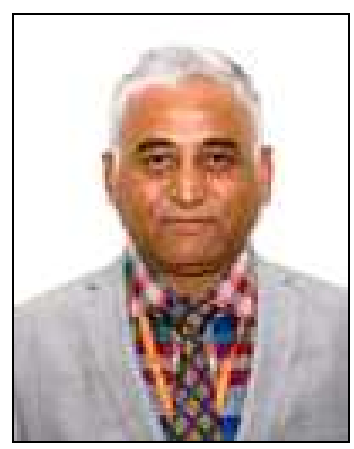

Prabhugoud I. Basarkod is a Professor at the School of Electronics and Communication Engineering, REVA University. His research areas include wireless networks, mobile adhoc networks (MANETs), agent technology, multimedia communication, and wireless sensor networks. He has 32 years of teaching experience with many research publications in reputed national/international journals and conference papers. He is a member of the Indian Society for Technical Education, Institute of Electrical and Electronics Engineers (IEEE), Member Institution of Electronics and Member of Institution of Electronics and Telecommunication Engineers (IETE).

E-mail: basarkodpi@ reva.edu.in

School of Electronics and Communication Engineering

REVA University

Bengaluru, India 